\title{
Parallel Performance of Declarative Programming using a PGAS Model
}

\author{
Rui Machado ${ }^{1,2}$, Salvador Abreu ${ }^{2}$, and Daniel Diaz ${ }^{3}$ \\ 1 Fraunhofer ITWM, Kaiserslautern, Germany \\ rui.machado@itwm.fhg.de \\ 2 Universidade de Évora and CENTRIA, Portugal \\ spa@di.uevora.pt \\ 3 University of Paris 1-Sorbonne, France \\ Daniel.Diaz@univ-paris1.fr
}

\begin{abstract}
Constraint Programming is one approach to declarative programming where a problem is modeled as a set of variables with a domain and a set of relations (constraints) between them. Constraint-based Local Search builds on the idea of using constraints to describe and control local search. Problems are modeled using constraints and heuristics for which solutions are searched, using Local Search. With the progressing move toward multi and many-core systems, parallelism has become mainstream as the number of cores continues to increase. Declarative programming approaches such as those based on constraints need to be better understood and experimented in order to understand their parallel behaviour. In this paper, we discuss experiments we have been carrying out with Adaptive Search and present a new parallel version of it based on GPI, a recent API and programming model for the development of scalable parallel applications. Our experiments on different problems show interesting speed-ups and, more importantly, a better understanding of how these gains are obtained, in the context of declarative programming.
\end{abstract}

Keywords: Constraint Programming, Local Search, Parallel Programming

\section{Introduction}

There is an inevitable paradigm shift towards multicore technologies where parallelism is now omnipresent. In recent systems, parallelism spreads over several systems levels and heterogeneity is growing on the node as well as on the chip level. Data must be maintained across a hierarchy of memory levels and most applications and algorithms are not ready to take full advantage of the available capabilities.

Parallel programming is usually a difficult and error-prone task. Although MPI [11] has become the de facto standard for parallel programming, there has been a demand for programming models with a flexible threads model and asynchronous communication. PGAS (Partitioned Global Address Space) programming models have been emerging as a valid alternative to MPI. 
One of the great features of declarative programming approaches is their potential simplification of the development of parallel programs, relieving the programmer from error-prone aspects related to explicit control, which can be very difficult to handle with parallel programming, while retaining enough expressive power to model complex real-world problems. One declarative approach is Constraint Programming: a problem is modeled as a set of variables over some domain and a set of relations (constraints) is required to hold between them. Program execution consists in finding a solution to (i.e., solving) the stated constraint problem. The solving process can use different methods, one of which is Local Search where, instead of exploring the complete search space, heuristics are used to guide the search to portions of the search space where solutions are more likely to be found. Local Search is based on the simple idea of "searching" by iteratively moving from one candidate solution to one of its neighbours. Despite its simplicity and effectiveness to handle hard problems, in order to solve large problem instances, parallelism should be introduced to cope with the large running time.

Our general aim is not only to simplify the use of parallelism of current systems with a declarative approach based on constraints but, at the same time, exploit that parallelism to tackle large and difficult problems.

We have been developing parallel designs for both complete (propagationbased [14]) and local search constraint solvers. This article reports on the latter.

The contribution of this paper is twofold: a new parallel design for the Adaptive Search method based on a PGAS Model and a better understanding of its parallel behaviour, easily extended to Local Search algorithms in general. We present and evaluate our new design based on GPI, showing interesting speed-up gains on benchmarks known to have scalability issues. We discuss the results and provide a deeper interpretation of the parallel behaviour of Adaptive Search in particular and of Local Search methods in general, based on some characteristics of the benchmarks.

The rest of the paper is organized as follows: in section 2 we present GPI and its programming model, hightlighting its important features. Section 3 provides some background on the Adaptive Search algorithm and section 4 focuses on its parallelization. In section 5 , we detail our parallelization strategy based on GPI and in section 6 we show the obtained results and compare it to the previous implementation. Section 7 examines and interprets our experimental findings, correlating them with the characteristics of the problems. Finally, section 8 presents a short conclusion and perspectives of future work.

\section{GPI}

The Partitioned Global Address Space (PGAS) is a parallel programming model which has been seen as a good alternative to the established MPI. The PGAS approach offers the programmer an abstract shared address space model which simplifies the programming task and at the same time facilitates data-locality, thread-based programming and asynchronous communication. GPI (Global ad- 
dress space Programming Interface) $[9]^{4}$ is a PGAS API for parallel applications running on clusters. The thin communication layer delivers the full performance of RDMA-enabled ${ }^{5}$ networks directly to the application without interrupting the CPU. Fig. 1 depicts the architecture of GPI.

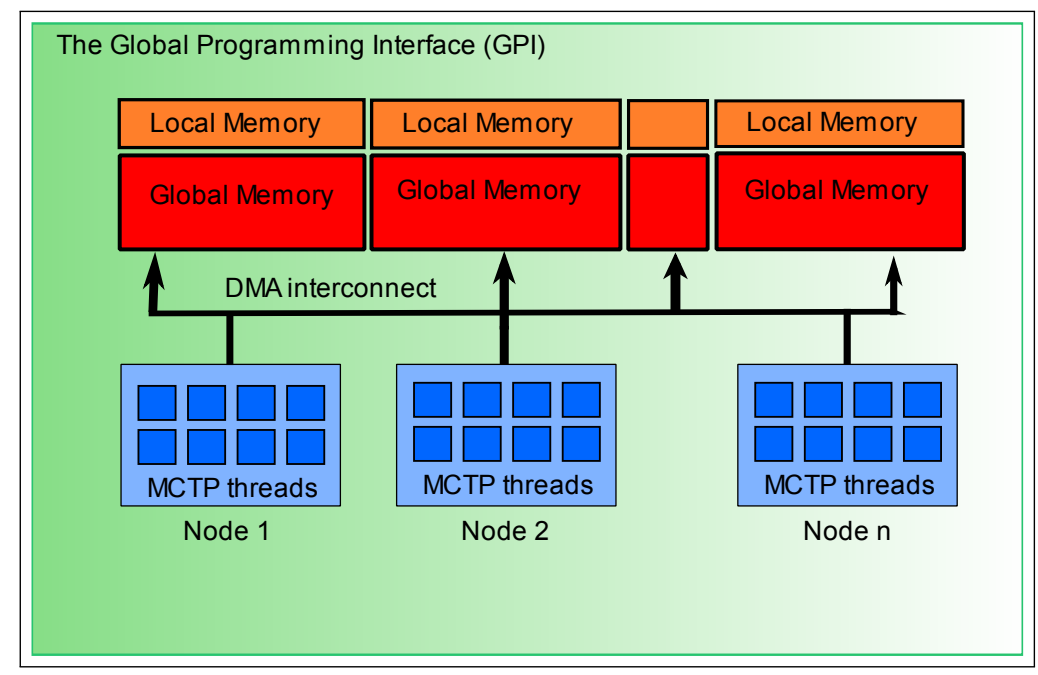

Fig. 1. GPI

The local memory is the internal memory available only to the node and allocated through typical allocators (e.g. malloc). This memory cannot be accessed by other nodes. The global memory is the partitioned global shared memory, available to other nodes, and where shared data should be placed. The DMA interconnect connects all nodes and is the underlying mechanism for most GPI operations. On each node, the Manycore Threading Package (MCTP) is used to take advantage of all cores present on the system. MCTP is a threading package based on thread pools that abstracts the native threads of the platform and a component of GPI.

The GPI core includes different functionalities but in the context of this work, the most important functionality is the read/write of global data.

Two operations exist to read and write from global memory independent of whether it is a local or remote location. One important point is that those operations are one-sided that is, only the peer that issues such operation takes part in it. This is different from a two-sided scheme (message passing) where the peer that sends (sender) has a corresponding peer (receiver) that needs to issue a receive operation. Moreover, this functionality is non-blocking and completely off-loaded to the interconnect, allowing the program to continue its execution

${ }^{4}$ GPI was previously known as Fraunhofer Virtual Machine (FVM).

${ }^{5}$ RDMA - Remote Direct Memory Access. 
and hence take better advantage of CPU cycles. The data movement does not require any intermediate buffers and protocols to maintain those buffers. If the application needs to make sure the data was transferred (read or write), it needs to call a wait operation that blocks until the transfer is finished and asserting that the data is usable.

\section{Adaptive Search}

Local Search is based on the simple idea of "chasing" a solution by iteratively moving from one candidate (call this a "configuration") to one of its neighbours. The neighbourhood of a configuration is the set of configurations that can be obtained by applying a move. A move is a local change (hence the name Local Search).

The mechanism used to select a neighbour and thus the definition of what constitutes a neighbourhood is the main issue that differentiates between different local search methods. In general, it is problem dependent and is related to the definition of the objective function.

The Adaptive Search method [4] is one of many different local search methods and has proved to be very efficient in the types of problems where it was tested. It is a generic, domain-independent constraint-based local search method.

This meta-heuristic takes advantage of the structure of the problem in terms of constraints and variables and can guide the search more precisely than a single global cost function to optimize, such as for instance the number of violated constraints. The algorithm also uses a short-term adaptive memory in the spirit of Tabu Search [12] in order to prevent stagnation in local minima and loops. This method is generic, can be applied to a large class of constraints (e.g. linear and non-linear arithmetic constraints, symbolic constraints, etc) and naturally copes with over-constrained problems.

The input of the method is a problem in CSP format, that is, a set of variables with their (finite) domains of possible values and a set of constraints over these variables. For each constraint, an "error function" needs to be defined; it will give, for each tuple of variable values, an indication of how much the constraint is violated. For instance, the error function associated with an arithmetic constraint $|X-Y|<c$, for a given constant $c \geq 0$, could be $\max (0,|X-Y|-c)$.

Adaptive Search relies on iterative repair, based on variable and constraint error information, seeking to reduce the error on the worst variable so far. The basic idea is to compute the error function for each constraint, then combine for each variable the errors of all constraints in which it appears, thereby projecting constraint errors onto the relevant variables. Finally, the variable with the highest error will be taken and its value will be modified. In this second step, the well known min-conflict heuristic is used to select the value in the variable domain which is the most promising, that is, the value for which the total error in the next configuration is least. In order to prevent being trapped in local minima, the Adaptive Search method also includes a short-term memory mechanism to store variables to avoid (variables can be marked Tabu and "frozen" for a number 
of iterations). It also integrates reset transitions to escape stagnation around local minima. A (partial) reset consists in assigning fresh random values to some variables (also randomly chosen). A reset is guided by the number of variables being marked Tabu. As in any local search method, it is also possible to restart from scratch when the number of iterations reaches a given limit.

\section{Parallel Adaptive Search}

When parallelizing an algorithm one aims at identifying hotspots and sources of parallelism. As with most meta-heuristics, in Adaptive Search these sources of parallelism are essentially: (1) the inner loop of the algorithm i.e., computing and combining the errors of variables and selecting the variable with highest error and (2) the search space of the problem.

The problem with exploiting the inner loop of the algorithm is its granularity: it is too fine-grained and the associated overhead might come at a too high cost.

The second main source of parallelism is the search space (domain) of the problem itself. Theoretically, this domain could be decomposed into several disjoint partitions, to be explored in parallel and without dependencies. However, in practice several issues arise with this: each partition is in general still too large for a sequential execution and, more importantly, the search space is not uniformly valid and the exploration should avoid areas that are known to lead to poor solutions. Moreover, it is hard and expensive to control and maintain the search conducted in the different partitions since a Local Search algorithm only has a local view of the search space. One example is the class of problems that have the best solutions clustered in a certain 'zone' of the search space. In this case, the algorithm should converge to that zone but in case of parallel execution avoid too much redundant work.

The Adaptive Search method has already been subject to some research on its parallel behaviour. Previous work on parallel implementations of the Adaptive Search algorithm have mostly focused on independent multiple-walks. Recal that independent multiple-walks are the simplest approach to parallel local search. A walk is carried out by each processor without any communication between them. Processors (search threads) start at a different solution and perform their own walk, intersecting or not, with walks from other processors. The same or different algorithms can be used to perform the walk, with the same or different parameters.

In [6], the authors present a parallel implementation of the Adaptive Search algorithm for the Cell/BE, a heterogenous multicore architecture. The system includes 16 processors (the $\mathrm{SPEs}^{6}$ ) where each one starts with a different random initial configuration. The $\mathrm{PPE}^{7}$ acts as the master processor, waiting for the message of a found solution. For this number of processing units, the results were very promising, achieving linear speed-up for most problems.

\footnotetext{
${ }^{6}$ Synergistic Processing Element

7 Power Processor Element
} 
Further work with Parallel Adaptive Search continued to follow the same approach with no communication between workers, but more interestingly, concentrating on cluster systems with a larger number of cores.

In [2], the authors experiment and investigate the performance of a multiple independent-walk search on a system with up to 256 cores. The parallelization was done with MPI and involves the introduction of a "communication step" which tests if termination was detected (a solution was found) and terminates the execution properly. The results are relatively modest in terms of parallel efficiency, far for the ideal speed-up, which is in contrast with the results obtained at a smaller scale (on the Cell/BE, ie. with up to 16 cores). This points out the need for better alternative strategies in order to better exploit large-scale parallelism.

Since the independent multiple-walk approach still leaves space for improvement in terms of parallel efficiency and scalability for some problems, new ways to take full advantage of parallel systems must be found.

In [1], the authors experiment with more complex strategies, where processes exchange messages resembling branch-and-bound methods where the bound is exchanged between all participants. In their work, two alternatives are attempted: exchanging the cost of the current configuration of each process and the current cost plus the number of iterations needed to achieve that cost. Unfortunately, neither approach achieves better results than an independent multiple-walk.

\section{Adaptive Search with GPI}

Previous work with parallel Adaptive Search provides some groundwork to build upon and has shown that some benchmarks exhibit scalability problems when run on a large number of cores.

GPI appears to be, à priori, an interesting match to the problem of parallelizing Adaptive Search: local search methods work with local information, trying to progress and converge on solutions in a global search space, requiring little global information. However, as demonstrated by previous work, some problems exhibit low parallel efficiency and communication and cooperation becomes requirements to obtain good scalability. The communication with GPI is based on one-sided primitives that ought to benefit the local view on a global search space, as it allows threads to cooperate asynchronously. Moreover, communication is very efficient as GPI exploits the full performance of the interconnect with little or no CPU intervention. Hence, we continue to explore ways to further improve the parallelization of the Adaptive Search algorithm, exploiting GPI and its programming model, with the objective of getting some further benefits. But more importantly, to find mechanisms, concepts or limitations that are general.

In general, we can define the following objectives:

- further investigate and understand the behaviour of parallel Adaptive Search on different problems. 
- investigate the possibilites given by GPI and devise more sophisticated mechanisms for the parallel execution of Adaptive Search, improving its performance

- identify the, possibly new, problems generated by the previous point.

The new parallel version of Adaptive Search based on GPI includes two variants which we name TDO (Termination Detection Only) and PoC (Propagation of Configuration).

The TDO variant implements the simple independent multiple-walk and serves mostly has our basis for comparison. First, with the existing MPI version, making sure that the implementation is correct and the performance is as expected. Second, to allow us to measure the improvement (if any) obtained with the more complex $\mathrm{PoC}$ variant. The $\mathrm{PoC}$ variant introduces more communication and sharing between working threads, by means of GPI primitives and its threaded model, but it is our expectation that this overhead will be offset by the performance gain.

The next sections present the two different variants in more detail.

\subsection{Termination Detection Only}

The variant with Termination Detection Only (TDO) is straightforward and implements the idea of independent multiple-walks: all available cores execute the sequential version of the Adaptive Search algorithm.

We name this variant "Termination Detection Only" because it amounts to a termination detection problem i.e., detecting the termination of a distributed computation. Termination Detection is in itself a subject of much research and several algorithms have been and continue to be proposed( $[7,10,15])$.

In the case of the Parallel Adaptive Search method, we are interested in detecting termination as soon as one of the participating threads has found a solution: we want to get the first (earliest) solution. The implementation of this variant is simple as it only requires a triggering mechanism.

The GPI implementation follows a line similar to the previous work with MPI. Whenever a thread finds a solution, it triggers termination by writing to its peers that it has found a solution. Thus, the wall-clock time of the parallel execution is the time taken by this fastest thread.

Other threads must detect termination. This entails introducing a communication step in the internal loop of the Adaptive Search algorithm. This is required since there is no other way for a GPI instance to react on an remote event (i.e., termination) other than with communication. In this step, a check for termination is done on a particular memory address that is written on termination emission as described above. The communication step introduces some overhead that needs to be kept low, thus it is only executed every $k$ iterations.

\subsection{Propagation of configuration}

The experiments in previous work and with the TDO variant have found that the simple approach to parallelization, namely, the independent multiple-walk, is 
insufficient to obtain parallel efficiency on some problems especially when experimenting with a large number of cores. Moreover, exchanging simple information such as the cost leads to no improvement. This result goes to show that this is not a reliable metric, at least not by itself: it just says that $\operatorname{cost} C$ (better than the current cost) can be achieved but says nothing about when and how to reach it.

Hence, we aim at communicating more, and more meaningful information, introducing cooperation. By cooperation we mean mechanisms that allow threads to share information about their state and thus benefit from the collective search. Also, we would like to exploit the potential and benefits of GPI and its programming model (one-sided communication, no wait for communication, global access to data, threaded model, etc.) This can be achieved, for instance, by moving towards algorithms which resort to more communication than in previous cases.

One of the most powerful aspects of Local Search is its simplicity. Because of this, it is not obvious what could be considered as the meaningful information to be shared and communicated to other threads. One promising candidate which hasn't yet been tried is the whole current configuration. ${ }^{8}$ The final configuration represents the solution when the algorithm stops.

The current implementation of the Adaptive Search method deals only with permutation problems and thus, a configuration is the permutation vector of the problems' variables.

Similarly to other approaches to parallelization which introduce cooperation, several important questions arise, namely:

1. Who does the communication?

2. When to do the communication?

3. How to do the communication?

4. What to communicate?

Our approach, which we call Propagation of Configuration (PoC), aims at answering these questions and giving a better understanding of how cooperation can help with increasing the scalability of Local Search in general and the Adaptive Search method in particular.

\section{Who does the communication?}

Note that on each node, there are as many threads as the number of availble cores. Communication is performed between nodes, by reading or writing the global memory of GPI. Hence, to answer this question we consider if, for each node, all or only a single thread actually performs communication with the other nodes.

There are potential advantages and disvantages with both options. If all threads perform communication, any shared resources must be protected by a mutual exclusion mechanism, which might suffer from high contention. Moreover,

\footnotetext{
${ }^{8}$ Because the term solution is sometimes misleading, we refer to the current solution as a configuration.
} 
when all threads perform communication, a lot more pressure on the interconnect follows, increasing the parallel overhead and with possibly a lot of redundant communication happening (the same configuration being passed around several times). On the other hand, there will be a rapid progress towards the best promising neighbourhood, intensifying the search. Of course, this can be positive but can also become dangerous since most of threads might get trapped in a local minimum or poor quality neighbourhood. A good trade-off between intensification and diversification needs to be achieved.

If a single master thread communicates, the effects are potentially the opposite: less intensification but also less contention, less pressure on the interconnect and less redundant work.

Preliminary tests have made it clear that the best option is the one with a single communicating thread since it reduces the parallel overhead. Moreover, with GPI, all threads in a single node benefit immediately from the results obtained by the master thread without any messages exchange.

\section{When to do the communication?}

The first possible answer to this question is to follow the same strategy as with the Termination Detection Only variant: introduce a communication step and perform communication every $k$ iterations. The value of $k$ has a very significant impact on performance: with a low value (e.g., $k=10$ ), a strong intensification of the search is achieved but with the danger that threads might give up too soon on a promising neighbourhood. With a high value of $k$, we avoid that danger but less intensification will be achieved since less information will be propagated.

The other option is to not interrupt the normal flow of the algorithm for communication, letting the search progress normally and independently until a local minimum is achieved. Only at this point the configuration is propagated and possibly used. One danger, however, is if threads do not hit local minima that often, the propagation of configuration will not progress and some threads might never see an up-to-date configuration. A solution to this problem is to still have communication every $k$ iteration, where threads only use the propagated information when they are "in trouble" i.e., they hit a local minimum. However, this option increases the overhead by adding the extra communication step in some iterations.

In principle the second option might seem more promising as no disturbance is caused when the algorithm is progressing well. But the aforementioned danger that the propagation of configurations won't progress can have the consequence that there won't be any benefit from the communication scheme when compared to the simple TDO variant. Prelimary tests on a problem with low number of local minima (Magic Squares) confirmed this fact. Hence and based on this reasoning, we opted to have a communication step. Our PoC variant combines termination detection and the propagation of configurations in a single step that happens every $k$ iterations and we focus the experimentation on finding an good value for $k$. 


\section{How to do the communication?}

With this question, we consider a single alternative. Since we aim at large scale executions (hundreds to thousands of nodes), we need an efficient approach. Communication is done along a tree-based topology, where each node only communicates with its parent and children (if any). Currently, a binary tree is used but this can be parametrized at initialization. At each communication step, the propagation of the configuration is done either up (to parent) or down (to the children) the tree. This only happens if a configuration was propagated from the children (in case of the up direction) or from the parent (down direction). The propagation of the communication behaves then like a wave, up and down the tree, with possibly different configurations being propagated at different points of the tree and contributing to some diversification.

Communication is performed by using GPI one-sided primitives. A thread posts a write operation and returns immediately to work. The configuration to be propagated will be directly written to the memory of the remote node, asynchronously, without any acknowledgement and overlapped with the algorithm's computation. The remote node on the other hand, on its communication step, checks if a valid configuration was written to its memory, decides how to act on it and propagates its decision further.

We consider this single alternative since it gives us a good balance between intensification and diversification and because having a tree-based topology provides an efficient pattern to achieve communication scalability, with good locality. The final objective is to have a communication step with low overhead and here GPI provides us with mechanisms to do so.

\section{What to communicate?}

The Adaptive Search method (as many other Local Search methods) is very simple and includes very few elements that can be communicated.

The proposed option has been already mentioned: to communicate a full configuration. To this, we only add the cost of the configuration as it is the metric to evaluate the configuration, and we need only compute it once.

Still, the question remains of which configuration to communicate. In our design the best configuration (with better cost) is communicated. At a communication step, a thread decides whether to propagate its own current configuration or the propagated configuration(s) it received from its neighbour(s).

Communicating configurations is advantageous because configurations implicitly contain more information about the global state of the search: as the best configurations are being propagated, threads that are currently on poorer neighbourhoods might benefit from moving to the best ones. With the stochastic behaviour of Adaptive Search and enough diversification, the whole search procedure can be performed on the best neighbourhoods and, hopefully, converge faster onto good solutions. 


\section{Experimental Results}

In this section we present the obtained results using a few benchmark problems.

- costas-array: the Costas Array problem [5],

- all-interval: the All Interval Series problem (prob007 in CSPLib [8]),

- magic-square: the Magic Square problem (prob019 in CSPLib).

The experiments were conducted on a cluster system where each node includes a dual Intel Xeon 5148LV "Woodcrest" (i.e., 4 CPUs per node) with 8 GB of RAM. The full system is composed of 620 cores connected with Infiniband (DDR). We performed our experiments on the system using up to 256 cores on some problems and 512 cores on others. The difference is due to the fact that the system is heavily in use and it is hard to get access to the full cluster.

Note that Adaptive Search, as many other Local Search methods, has a stochastic behaviour to achieve diversity on the search. To benchmark such behaviour, several executions must be done and averaged. In our experiments we ran each problem 100 times in order to obtain meaningful results.

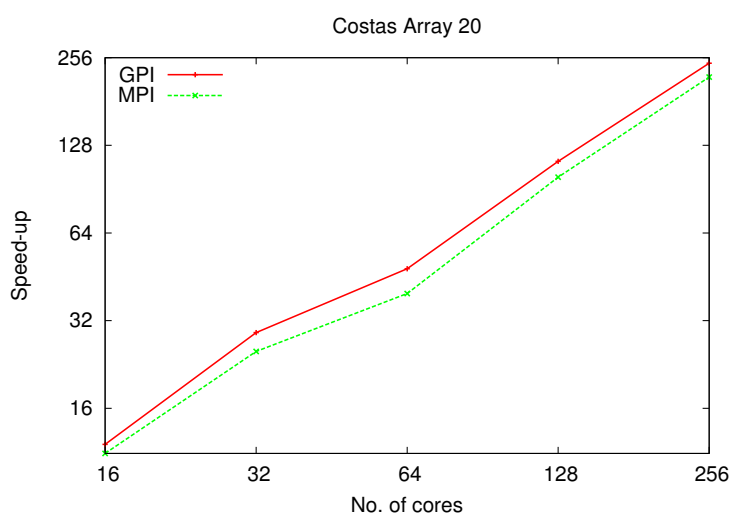

Fig. 2. $\mathrm{CAP}(20)$ on 256 cores (64 nodes)
We compare both GPI variants (TDO and PoC) with the MPI implementation, as a basis for comparison. Figure 2 depicts the obtained results for the Costas Array problem (CAP) with $n=20$.

As already observed in [3], the CAP shows an almost optimal scalability using an independent multiple -walk with no cooperation. We can observe that our implementation obtains similar, although slight better, results. This is

the expected result since both approaches (TDO and MPI) are equivalent: communication is only used for detecting termination. Nevertheless, it is a confirmation that our implementation performs as expected.

Although we aspired at obtaining even better results with the $\mathrm{PoC}$ variant (possibly super linear) for this problem, our experiments showed that this variant performs much worse than the simple TDO variant and thus we only present the speedup obtained with GPI using the TDO variant.

Figure 3 depicts the obtained results for the Magic Square problem up to 512 cores. For this problem we present the speedup obtained with the TDO and PoC variants and compare it with the MPI version. The GPI TDO variant presents again, as expected, results similar to the MPI version. 


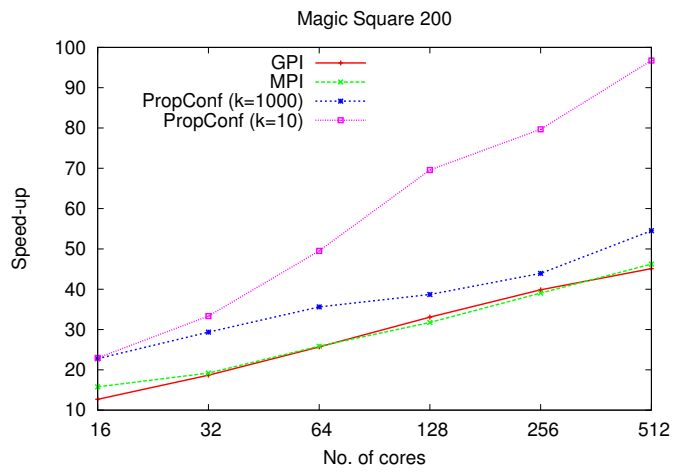

Fig. 3. MS(200) on 512 cores (128 nodes)

The Magic Square problem is one of the problems that results in disappointing scalability when using the simple independent multiplewalk and therefore a major target for improvement with more sophisticated approaches. Indeed, for this problem, our PoC variant improves the performance and scales better as we increase the number of cores used.

We wanted to answer the question of when to do communication: as we mentioned, in our preliminary experiments it turned out that the best approach is to have a communication step every $k$ iterations where the value of $k$ is decisive. Surprinsingly, for this problem, a lower value of $k(k=10$ in contrast to $k=1000)$ improves scalability by a factor of 2 , achieving a speedup of 97 with 512 cores. Still a low parallel efficiency but a very significant improvement over the other options and variants.

The results we obtained for the last problem, the All Interval series $(n=400)$, are shown in Figure 4.

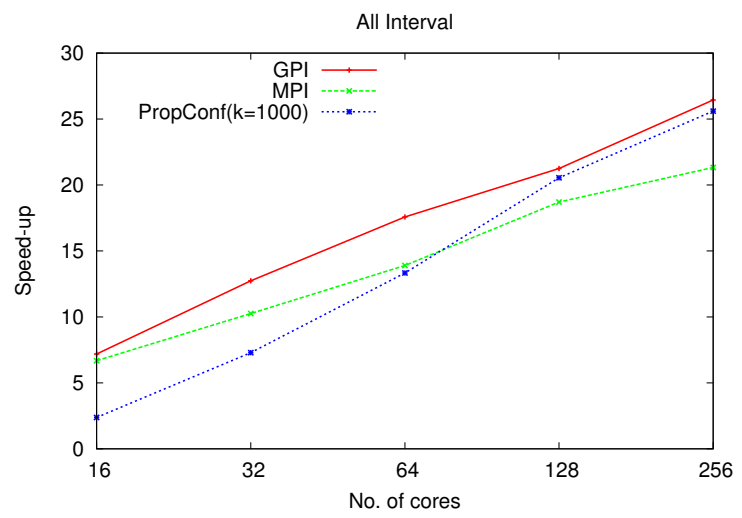

Fig. 4. $\mathrm{AI}(400)$ on 256 cores (64 nodes)
The All Interval Series benchmark is also one of the problems where good scalability was hard to reach when using a large number of cores. In Figure 4, one may observe this fact where both the MPI and GPI TDO versions reach a modest speedup factor of 20 and 25, respectively (with 256 cores). Our PoC variant however, performs much worse than the TDO variant at a low number of cores but it improves as we increase the

number of cores, hinting that this variant can be of advantage if we increase the number of cores and the problem size. In Fig. 4 we only depict the obtained results for the $\mathrm{PoC}$ variant with $k=1000$ since, for this benchmark, it is the best value. In contrast to the Magic Squares benchmark, a lower value of $k$ results in a much worse performance. 


\section{Discussion}

The experimental results presented large differences in how the different problems benefit from parallelism and the implemented variants. One of our main objectives is to investigate and understand why this happens.

In order to be able to draw some conclusions on our experiments, it is important to characterize the chosen problems from different perspectives. We do so, resorting to different information such as the number of iterations and local minima. This characterization will give us a basis to better understand the problems at hand and ultimately explain our results.

Table 1 presents the obtained values for acquired information when running some instances of the previously presented problems. This information is the following:

Problem The problem instance.

Iterations The number of iterations required to find a solution.

Local Minima The number of local minima found.

Resets The number of partial resets performed (not full restart).

Same var / Iteration The number of times there was more than one candidate variable (highest error value) to be chosen from.

This information allows us to better understand how does the Adaptive Search algorithm progress towards a solution, the neighbourhood structure and extract further information (e.g., number of local minima per iteration).

\begin{tabular}{|c|r|r|r|r|}
\hline Problem & Iterations & $\begin{array}{r}\text { Local } \\
\text { Minima }\end{array}$ & Resets & $\begin{array}{r}\text { Same var } \\
\text { Iteration }\end{array}$ \\
\hline \hline Magic Square 200 & 413900 & 25864 & 3 & 23.36 \\
\hline Costas 18 & 389932 & 204024 & 204024 & 1.00 \\
\hline Costas 19 & 3364807 & 1714299 & 1714299 & 0.99 \\
\hline All Interval 200 & 11229 & 495 & 495 & 5.97 \\
\hline All Interval 400 & 41122 & 1422 & 1422 & 9.19 \\
\hline
\end{tabular}

Table 1. Information collected for different problems instances.

From Table 1 we can see that the different problems exhibit a significantly different behaviour. Magic Square performs a low number of partial resets when compared to the total number of iterations or to the number of identified local minima. On the other hand, it is the problem where the number of candidate variables per iterations (Same var/Iteration) is highest, meaning that at each iteration there are several possible moves towards the next configuration.

The Costas Array problem exhibits a completely different behaviour. In this case, the number of identified local minima is very large (almost every second 
iteration finds a local minimum) and the number of partial resets is also very high, coincident with the number of local minima i.e., at each local minimum found, a partial reset is performed. Also the number of possible moves at each iteration is close to 1 .

All Interval is yet another kind of problem. Here, the number of resets is as with the CAP equal to the number of local minima but these happen much less often. The number of possible variable choices or moves is higher than 1 , meaning that some diversification could be achieved.

If we relate this characterization of problems with the obtained experimental results, some conclusions can be drawn in order to better understand the parallelization behaviour of this algorithm or, more concretely, how much can it benefit from a communication scheme such as the one we designed.

We argue that one critical aspect is the density of the neighbourhood of a configuration or the set of possible moves, which define transitions between configurations. Since we are propagating configurations we can look at our problems at hand according to this aspect. If a problem has a dense neighbourhood or, in other words, the set of possible moves at each transition is (much) larger than one, each of these moves can be explored in parallel. Thus, when a promising configuration is propagated and several moves are possible and explored in parallel, the probability that one of these moves leads to a faster path towards an optimal solution increases.

Another important aspect is the number of local minima and resets and how both relate. A problem that finds a large number of local minima before encountering an optimal solution benefits less from processing a configuration which seems promising. This configuration is heuristically promising but in reality this information is less meaningful than it should. Similarly, a problem with a high number of partial resets suffers from the same problem.

Looking back at our experimental results with the different problems, we can better understand a) the difference in scalability and b) the improvement factor brought by the PoC variant to some problems.

In the Magic Square problem, each configuration has a dense neighbourhood and benefits from the parallel exploration of different moves. Thus, the $\mathrm{PoC}$ variant improves the performance and scalability of the algorithm. When a working thread adopts a propagated configuration, it will define its own path from that configuration and differently from one other thread that receives that same promising configuration. Moreover, this problem has a low number of local minima and resets meaning that paths from one (initial) configuration towards an optimal solution are a series of transitions from neighbour configurations.

The Costas Array Problem exhibits optimal scalability with the independent multiple-walk MPI version or with our TDO variant and this is already per se satisfactory. On the other hand, it performs worse with the $\mathrm{PoC}$ variant: propagating a configuration is only a source of parallel overhead and will limit the search allowing less diversification. A propagated configuration will allow, on average, a single move and two threads taking the same configuration results in redundant work. This is also probably unfruitful since the CAP is one of the 
problems with a high number of local minima and resets. This also explains the good scalability using the TDO variant, where increasing the number of cores allows covering more of the total search space together with the fact that solutions for this problem are well spread over it.

Finally, the All Interval Series problem shows a mixed behaviour. Similarly to the CAP, the larger number of local minima found and same number of resets point to the same situation: there is less benefit from taking a propagated configuration since its meaningfulness is low. The PoC variant only introduces unnecessary overhead and this could explain the much worse performance at a lower number of cores. On the other hand, and similarly to the Magic Square benchmark, there is more than one possible move, on average i.e., some diversification can be achieved. With a large enough number of cores, the parallel overhead can be amortized by the gain obtained with this diversification. This could be the reason for the steeper curve for the PoC variant on Fig. 4. Of course, with further experiments we will be able to understand this better.

In summary, problems that follow a trajectory with a single possible move won't benefit from a communication scheme that propagates the best current configuration(s). Also, if a large number of local minima is found and partial resets are required in the same number, the expectation for improvement in performance is rather low. On the contrary, problems where configurations have a denser neighbourhood benefit from a cooperation scheme such as the $\mathrm{PoC}$ variant where the full configuration is communicated and improvements in performance are expected.

\section{Conclusion}

In this paper we presented our work on the parallel implementation of the Adaptive Search method using an alternative programming model. GPI is an API designed for high-performance and scalable parallel applications. We aimed at investigating and understanding the behaviour of Adaptive Search in a parallel setting, focusing on different problems particularly those that, in previous work, showed scalability problems when targeting a large number of cores. GPI and its programming model allowed us to design a new communication and parallelization scheme which in our experimental evalution allowed a gain of a factor of 2 in terms of speedup for some problems. More importantly, it provided deeper insight and understanding on the parallelization of Local Search methods given different problems with disparate characteristics such as the density of a configuration neighbourhood, the number of local minima and partial resets.

We point out that GPI performs well and allows us to adopt more communication -intensive schemes, which supports the claim that solving local search problems is a good use case for GPI.

In the future, we intend to examine our design and conclusions with other larger problems and experiment with more sophisticated parallelization schemes. One possible direction is, instead of using promising information (configurations, cost, statistics) directly, to act on its complement, avoiding redundant work 
and thereby cover as much as possible from the search space since this is the main source of parallelism. Another direction is to revisit the modeling of each problem knowing that it will be executed in parallel; this is relevant as the current models are designed and optimized for sequential execution. Models designed with parallelism in mind, even if less efficient in serial execution, will benefit at scale as more cores are used in the solving process.

One of our potential final goals is the design of a new Local Search algorithm based on Adaptive Search and more amenable to parallelization, building upon the experiences presented in this paper.

Ultimately, the work described herein will be integrated with MaCS, a GPI port of the PaCCS hierarchical distributed constraint solving system [13], providing additional insight on how to reach good parallel performance on CSPs.

Acknowledgements This work was partly supported by Fundação para a Ciência e Tecnologia under grant PTDC/EIA-EIA/100897/2008 (HORUS).

\section{References}

1. Yves Caniou and Philippe Codognet. Communication in parallel algorithms for constraint-based local search. In IPDPS Workshops, pages 1961-1970, 2011.

2. Yves Caniou, Philippe Codognet, Daniel Diaz, and Salvador Abreu. Experiments in parallel constraint-based local search. In Peter Merz and Jin-Kao Hao, editors, EvoCOP, volume 6622 of Lecture Notes in Computer Science, pages 96-107. Springer, 2011.

3. Yves Caniou, Daniel Diaz, Florian Richoux, Philippe Codognet, and Salvador Abreu. Performance analysis of parallel constraint-based local search. In Proceedings of the 17th ACM SIGPLAN symposium on Principles and Practice of Parallel Programming, PPoPP '12, pages 337-338, New York, NY, USA, 2012. ACM.

4. P. Codognet and D. Diaz. Yet another local search method for constraint solving. Stochastic Algorithms: Foundations and Applications, pages 342-344, 2001.

5. John Costas. A study of detection waveforms having nearly ideal range-doppler ambiguity properties. Proceedings of the IEEE, 72(8):996-1009, 1984.

6. Daniel Diaz, Salvador Abreu, and Philippe Codognet. Targeting the cell broadband engine for constraint-based local search. Concurrency and Computation: Practice and Experience, 24(6):647-660, 2012.

7. Edsger W. Dijkstra, W. H. J. Feijen, and A. J. M. van Gasteren. Derivation of a termination detection algorithm for distributed computations. Inf. Process. Lett., 16(5):217-219, 1983.

8. Ian P. Gent and Toby Walsh. Csplib: A benchmark library for constraints. In $C P$, pages 480-481, 1999. http://www.csplib.org.

9. Rui Machado and Carsten Lojewski. The fraunhofer virtual machine: a communication library and runtime system based on the rdma model. In Computer Science-Research and Development, volume 23(3), pages 125-132, 2009.

10. Friedemann Mattern. Algorithms for distributed termination detection. Distributed Computing, 2:161-175, 1987. 10.1007/BF01782776.

11. MPI Forum. MPI: A Message-Passing Interface Standard. Version 2.2, September 4th 2009. available at: http://www.mpi-forum.org (Dec. 2009). 
12. Panos M. Pardalos, Leonidas S. Pitsoulis, Thelma D. Mavridou, and Mauricio G. C. Resende. Parallel search for combinatorial optimization: Genetic algorithms, simulated annealing, tabu search and grasp. In proceedings of IRREGULAR, pages 317-331, 1995.

13. Vasco Pedro, Rui Machado, and Salvador Abreu. A Parallel and Distributed Framework for Constraint Solving. In Proceedings of the 1st Workshop on Parallel Methods for Constraint Solving (PCMS 2011), 2011.

14. F. Rossi, P. Van Beek, and T. Walsh. Handbook of constraint programming, volume 2. Elsevier Science, 2006.

15. Vijay A. Saraswat, Prabhanjan Kambadur, Sreedhar B. Kodali, David Grove, and Sriram Krishnamoorthy. Lifeline-based global load balancing. In PPOPP, pages 201-212, 2011. 